\title{
Multidetector computed tomography diagnosis of splenic flexure volvulus: usefulness of multiplanar reformats
}

Sharon Zhiling Koh ${ }^{1}$, MrCs, MMed, Albert Su Chong Low ${ }^{2}$, FRCR, FAMS, Hak-Mien Quah ${ }^{1}$, FRCSE, FAMS

ABSTRACT Splenic flexure volvulus is the least common form of colonic volvulus. Preoperative radiological diagnosis is usually made presumptively, based on plain radiographs and/or barium contrast enema study in patients presenting with acute large bowel obstruction. We report a case in which multidetector computer tomography findings were diagnostic of this condition, thus highlighting the usefulness of multiplanar reformatting.

Keywords: colon, multidetector CT, multiplanar reformats, splenic flexure, volvulus

\section{INTRODUCTION}

Colonic volvulus accounts for $2 \%-4 \%$ of large bowel obstruction, ${ }^{(1)}$ with those that arise from the splenic flexure being the least common (approximately $1 \%-3 \%$ of colonic segmental volvulus).(2) Splenic flexure volvulus was first reported in 1953 by Glazer and Adlersberg, followed by Buenger a year later. By 2006, only 38 cases of splenic flexure volvulus had been reported, with patients spanning the ages of 7-78 years, attesting to the rarity of this entity. ${ }^{(3)}$ In this case report, we discuss the presentation, diagnosis and subsequent management of splenic flexure volvulus in a 23-year-old patient. The diagnosis was made using multidetector computer tomography (MDCT), a newer radiologic technique that enabled multiplanar reformatting.

\section{CASE REPORT}

A 23-year-old woman presented with a three-day history of vomiting with central abdominal colic and distension. She had a history of chronic constipation and recurrent abdominal colic but no history of chronic laxative use or prior abdominal surgery. On examination, there was epigastric tenderness and distension without signs of peritonism. Digital rectal examination revealed brown faecal stains and no faecal impaction.

Plain abdominal radiograph showed a grossly dilated ascending and transverse colon, as well as a splenic flexure with a possible distal cut-off (Fig. 1). The colon distal to the cut-off appeared to be collapsed. Contrast-enhanced MDCT of the abdomen and pelvis with multiplanar reformatting demonstrated a grossly dilated, mainly air-filled colonic segment in the anterior portion of the central upper abdominal cavity. The colonic segment was in an inverted $U$-shaped configuration with convergence of the tapered ends of the limbs. Extending from this convergence posteriorly and to the left was a whirl sign, consisting of a collapsed segment of the distal colon and its

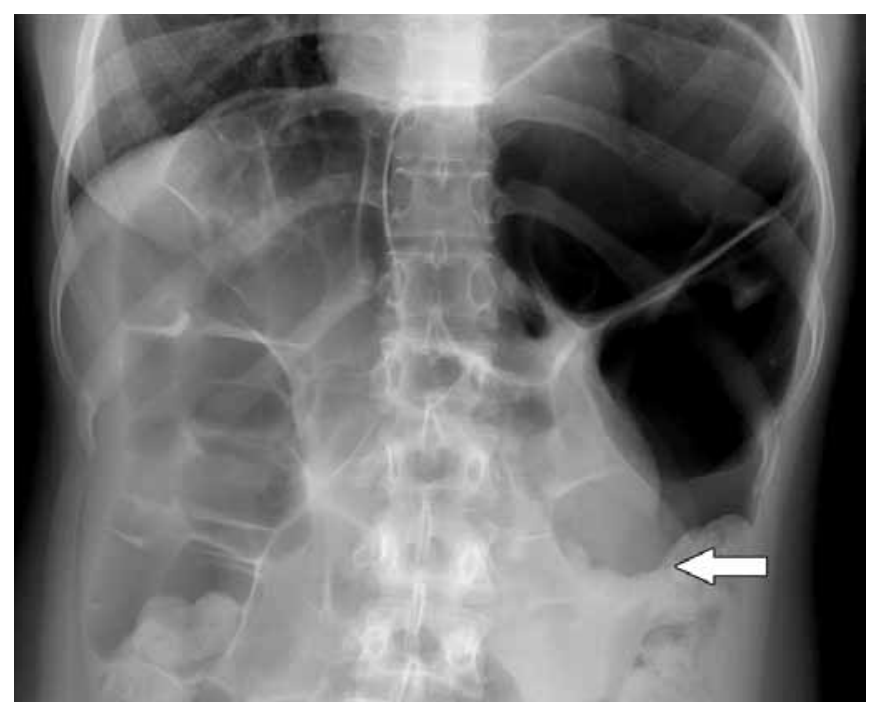

Fig. 1 Plain abdominal radiograph shows a grossly dilated ascending and transverse colon, and a splenic flexure with a possible distal cut-off (arrow).

attendant swirling branches, as well as tributaries of the left colic arteries and veins (Figs. 2a \& b). Adjacent to the whirl sign, a sharp-beaked appearance of the upper end of the descending colon, which had been retrogradely opacified with luminal contrast, is depicted (Fig. 2c). In other CT images (not shown), the dilated transverse colon could be traced proximally and was shown to course horizontally across the abdomen just inferior to the grossly dilated epigastric colonic segment. A normal splenic flexure could not be found. The stomach and spleen were in normal positions. These findings were entirely consistent with splenic flexure volvulus. There was no evidence of bowel perforation.

An optical colonoscopy showed megacolon and splenic flexure volvulus that had detorted. Eventually, the patient

${ }^{1}$ Department of Colorectal Surgery, Singapore General Hospital, ${ }^{2}$ Department of Diagnostic Radiology, Singapore General Hospital, Singapore

Correspondence: Dr Hak-Mien Quah, Consultant, Department of Colorectal Surgery, Singapore General Hospital, Outram Road, Singapore 169608. quahhm@colorectalcentre.com.sg 

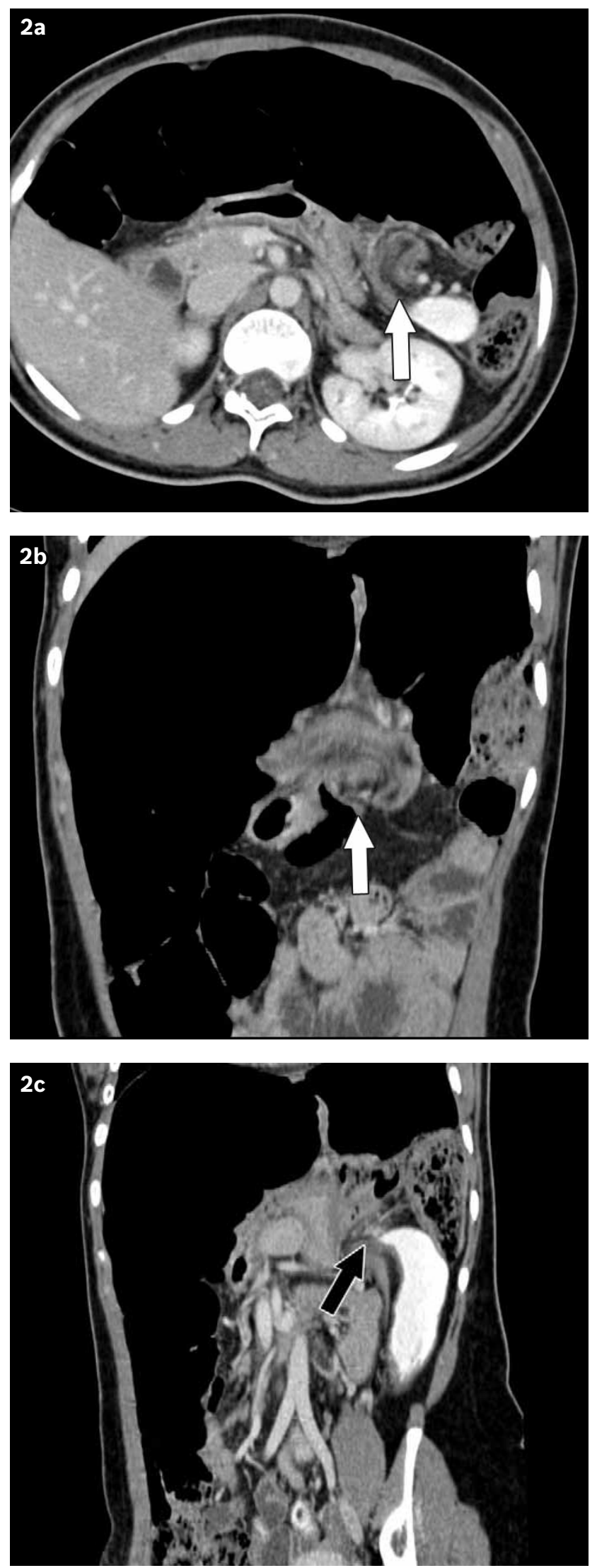

Fig. 2 (a) Axial and (b) coronal reconstructed contrast-enhanced CT images show a whirl sign (white arrows) consisting of an immediately distal collapsed segment of the colon and its attendant swirling branches, as well as tributaries of the left colic arteries and veins. (c) Coronal reconstructed contrast-enhanced CT image shows a sharp-beaked appearance of the upper end of the descending colon (black arrow), which had been retrogradely opacified with luminal contrast. The beak-like structure is adjacent to the whirl sign.

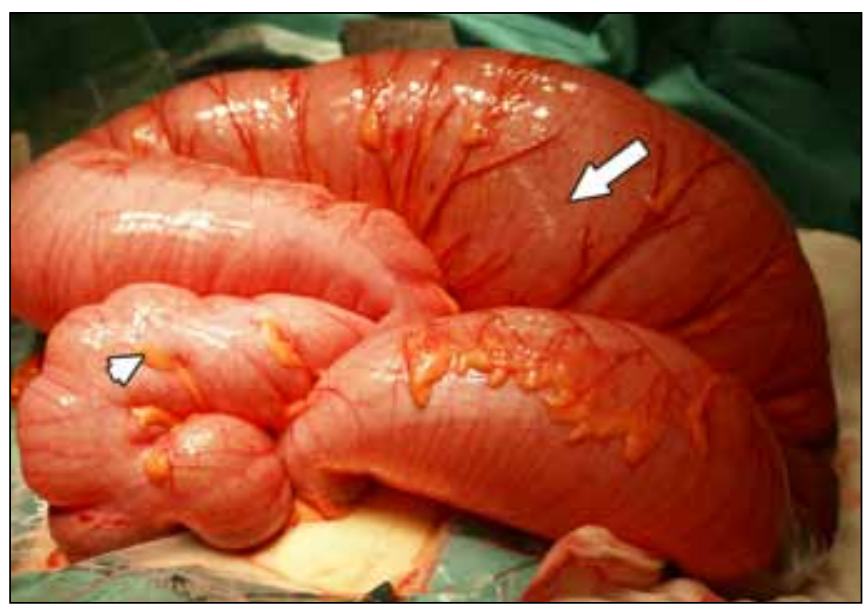

Fig. 3 Intraoperative photograph shows a grossly distended, redundant and mobile splenic flexure (arrow), with associated megacolon of the proximal colon (arrowhead).

underwent laparotomy, and a very long, redundant and grossly dilated splenic flexure with a long mesentery was found (Fig. 3). The rest of the colon was also dilated, with features of megacolon. There was no evidence of bowel gangrene, necrosis or congenital abnormalities. The usual anatomical fixation structures of the splenic flexure, i.e. the gastrocolic, phrenicocolic and splenocolic ligaments, were absent. Both the position and structure of the spleen and stomach were normal. The rectum was also normal. Total colectomy and primary ileorectal anastomosis were carried out. Histopathology showed a dilated colon with serosal congestion that was consistent with a recent episode of volvulus, as well as normal bowel muscle wall and neural plexus. No ischaemic or gangrenous segments were present. The patient made a full recovery from surgery. At follow-up three years post operation, she remained well and had regular bowel movements once or twice daily.

\section{DISCUSSION}

Volvulus of the colon is defined as an axial twist of a portion of the colon along its mesentery. The most common site of large bowel volvulus is the sigmoid colon, followed by the caecum. Splenic flexure volvulus is the rarest form of colonic volvulus, with less than 40 cases reported since 1953. It accounts for approximately $1 \%-3 \%$ of colonic segmental volvulus, and is slightly more common in females. ${ }^{(4)}$ The rarity of its incidence is due to the anatomical attachment of the splenic flexure to its peritoneal folds, i.e. the phrenicocolic, gastrocolic and splenocolic ligaments. ${ }^{(1)}$ The splenic flexure is usually immobile in the presence of these rigid ligamentous attachments. Congenital absence, surgical division or laxity of the abovementioned ligaments, as well as a long and redundant mesentery, can predispose an individual to splenic flexure volvulus. ${ }^{(5)}$

Associated dysmotility disorders, including pseudoobstruction, ${ }^{(6)}$ chronic constipation ${ }^{(2)}$ and triad syndrome, have been described over the last few decades. As described by Ballantyne, ${ }^{(2)}$ chronic constipation results in redundancy of the colon and elongation of the mesentery, which predisposes the 
affected individual to the formation of volvulus. A history of previous surgery leading to the disruption of natural ligaments in the left hypochondrial region of the peritoneal cavity, or the formation of adhesion bands that act as the axes of rotation, have also been reported to predispose one to formation of volvulus. (7)

The clinical presentation of splenic flexure volvulus is usually acute large bowel obstruction with associated features such as obstipation and gradual abdominal distention. There may be an associated history of previous abdominal surgery and/or chronic constipation. Hajivassiliou et $\mathrm{al}^{(8)}$ reported a case of a splenic flexure volvulus with symptoms and signs of proximal small bowel obstruction, which was diagnosed only during surgery. Osuka et $\mathrm{al}^{(3)}$ described a case involving a seven-year-old girl with a history of chronic idiopathic intestinal pseudo-obstruction syndrome (CIIPS). The authors suggested that signs of peritonism may arise when there is critical compromise of the blood supply to the affected colon. As in any type of mechanical intestinal obstruction, bowel ischaemia followed by gangrene, is the likely outcome if presentation or treatment is delayed. ${ }^{(3)}$

Suspicion of splenic flexure volvulus is often raised with plain radiographic findings of a markedly dilated, air-filled colon with an abrupt termination at the anatomic splenic flexure. A fluoroscopic contrast enema study may show a characteristic 'bird's beak' at the point where the colon is twisted. ${ }^{(1)}$ Most cases reported in the literature have based their preoperative diagnosis on the above two radiological modalities. There are, however, four typical radiological findings: a markedly dilated colon up to the area of the splenic flexure; two widely separated fluid levels in the caecum and transverse colon; an empty distal colon; and the characteristic 'bird's beak', as described above. ${ }^{(1)}$

On CT, specific features have been described for sigmoid and caecal volvulus, and descending colon volvulus. ${ }^{(9-11)}$ The features are mainly based on the presence of a whirl sign, which is composed of tightly twisted bowel, mesentery and vessels. CT is more specific than plain radiography and barium enema fluoroscopy, as it is able to precisely delineate the presence, cause, level and degree of volvulus, as well as detect bowel perforation, if any. In our case, the markedly dilated colon depicted on radiography was suggestive of volvulus and its position was indicative of the splenic flexure; our suspicion was confirmed on CT. The whirl sign at the ends of the convergent limbs of the dilated splenic flexure is diagnostic of splenic flexure volvulus. Furthermore, the beak-like appearance of the upper end of the descending colon, which correlates to the feature classically described on barium enema, as well as the absence of a normal splenic flexure, enabled us to make a precise CT diagnosis. In 2004, Ebihara et al ${ }^{(12)}$ described the use of 3D-CT in a 90-year-old patient with sigmoid volvulus. In this case, there was adequate visualisation of the central mesenteric vessels and the degree of colonic torsion could be assessed as well. The patient was managed using endoscopic decompression, as the authors were confident that the visualisation of the entire bowel in a single 3D image was able to reveal the presence and location of the obstruction, as well as allow the identification of vital potential complications such as perforation, ischaemia and adhesions. Management of the patient using endoscopic decompression was successful. ${ }^{(12)}$

Various treatment modalities for the management of this rare condition have been described. In order to prevent the recurrence of splenic flexure volvulus, two important points must be addressed, namely the acute problem involving the segment of the colon within the volvulus and the assessment of the rest of the bowel. The current treatment of choice for uncomplicated colonic volvulus is endoscopic decompression, which is especially appropriate in absence of rebound tenderness, peritoneal irritation and intestinal bleeding. However, this treatment is more applicable for sigmoid volvulus than splenic flexure volvulus.

The preferred primary definitive treatment for splenic flexure volvulus is surgical resection with end-to-end anastomosis. ${ }^{(13)}$ However, in an emergency situation, strict adherence to this recommendation may not be appropriate. Other methods described include detorsion of the involved bowel with colopexy, ${ }^{(14)}$ and decompression colostomy as a temporary measure. ${ }^{(2)}$ Resection with end colostomy and closure of the stump or mucous fistula is a safe surgical option, as described by Goldberg et al in 1984. ${ }^{(13)}$ The creation of stomas is reserved for patients who present with a gangrenous bowel that has perforation and intraperitoneal contamination. Nonsurgical endoscopic decompression or detorsion should be reserved only for extremely high-risk patients who are not suitable candidates for surgery. Otherwise, these procedures should be viewed as bridging or temporary measures prior to definitive surgical resection, as the rate of recurrence after detorsion alone is $20 \%-25 \%$. $^{(16)}$ Recurrence necessitates a second operation, which would expose patients to general anaesthesia, the risk of another bowel anastomosis, and if adhesiolysis is required, prolonged operative time with the inherent risk of serosal tears.

In our case, total colectomy was performed, as the patient had concomitant megacolon, which posed a risk for recurrent colonic volvulus if only limited colonic resection was performed. In 2004, Tobinaga et $\mathrm{al}^{(15)}$ concluded that it was unclear whether the concomitant occurrence of megacolon was a complication of volvulus or a coexisting condition. While some opine that megacolon will typically go into remission after the underlying disease has been treated, we recommend that resection be performed if conservative therapy fails, or if the signs and symptoms are suggestive of inadequate intestinal motility.

In conclusion, this report describes the constellation of $\mathrm{CT}$ features in splenic flexure volvulus, with the emphasis on the usefulness of multiplanar reconstruction, which depicts the various features of the condition, thus allowing precise preoperative diagnosis. Other features, such as the presence of megacolon, can enable better preoperative planning and counselling of the patient. 


\section{REFERENCES}

1. Mindelzun RE, Stone JM. Volvulus of the splenic flexure: radiographic features. Radiology 1991; 181:221-3.

2. Ballantyne GH. Sigmoid volvulus: high mortality in county hospita patients. Dis Colon Rectum 1981; 24:515-20.

3. Osuka A, Ikegami R, Watanabe Y. Splenic flexure volvulus in a child with chronic idiopathic intestinal pseudo-obstruction syndrome. Pediatr Surg Int 2006; 22:833-5.

4. Narasimharao KL, Mengi Y, Mitra SK, Pathak IC. Volvulus of the splenic flexure of the colon in a child. Aust N Z J Surg 1984; 54:385-6.

5. Cho YU, Sohn SK, Chi HS, Kim KW. Volvulus of the splenic flexure of the colon. Yonsei Med J 1994; 35:97-100.

6. Reinarz S, Smith WL, Franken EA Jr, et al. Splenic flexure volvulus: a complication of pseudoobstruction in infancy. AJR Am J Roentgeno 1985; 145:1303-4.

7. Young MO. Coexistent volvulus of splenic flexure and cecum. Surgery 1955; 37:983-90.

8. Hajivassiliou CA, Farrow G, Harvey J. Splenic flexure volvulus presenting as proximal small intestinal obsutrction. Aust N Z J Surg 1999; 69:318-9.

9. Shaff MI, Himmelfarb E, Sacks GA, Burks DD, Kulkarni MV. The whirl sign: a CT finding in volvulus of the large bowel. J Comput Assist Tomogr 1985; 9:410.

10. Frank AJ, Goffner LB, Fruauff AA, Losada RA. Cecal volvulus: the CT whirl sign. Abdom Imaging 1993; 18:288-9.

11. Chen A, Yang FS, Shih SL, Sheu CY. Case report. CT diagnosis of volvulus of the descending colon with persistent mesocolon. AJR Am J Roentgenol 2003; 180:1003-6.

12. Ebihara S, Okazaki T, Takahashi H, et al. Significance of three-dimensional computed tomography in a very old patient with sigmoid volvulus. Geriatr Gerontol Int 2004; 4:181-3.

13. Goldberg M, Lernau OZ, Mogle P, Dermer R, Nissan S. Volvulus of the splenic flexure of the colon. Am J Gastroenterol 1984; 79:693-4.

14. van Hasselt EJ. Volvulus of the splenic flexure and colon. Trop Doct 1995; 25:136-7.

15. Tobinaga S, Morinaga A, Sajima S, Kanazawa N, Yoshida T. Transverse to descending colon volvulus and megacolon with mesenterium commune: report of a case. Surg Today 2004; 34:875-7.

16. Hellinger MD, Steinhagen RM. Colonic Volvulus. In: Beck DE, Roberts PL, Rombeau JL, Stamos MJ, Wexner SD, eds. The ASCRS Manual of Colon and Rectal Surgery. New York: Springer, 2009: 409-26. 